\title{
Assessment of Respiratory Flow Cycle Morphology in Patients with Chronic Heart Failure
}

\author{
Ainara Garde • Leif Sörnmo • Pablo Laguna • \\ Raimon Jané • Salvador Benito • \\ Antoni Bayes-Genis • Beatriz F. Giraldo*
}

\begin{abstract}
This work investigates the significance of a number of time domain parameters for characterizing respiratory flow cycle morphology in patients with chronic heart failure $(\mathrm{CHF})$. The differences between these parameters are assessed considering the following three classification: CHF patients with periodic breathing (PB) vs $\mathrm{CHF}$ patients with non-periodic breathing (nPB), CHF patients (both $\mathrm{PB}$ and nPB) vs healthy subjects, and nPB patients vs healthy subjects. 26 CHF patients (8/18 with
\end{abstract}

A. Garde

Institut de Bioenginyeria de Catalunya (IBEC), Barcelona, Spain

E-mail: agarde@ibecbarcelona.eu

L. Sörnmo

Department of Biomedical Engineering and Center of Integrative Electrocardiology, Lund University, Lund, Sweden. E-mail: leif.sornmo@eit.lth.se

P. Laguna

Biomedical Signal Interpretation and Computational Simulation Group (BSICoS), Aragón Institute of Engineering Research (I3A), Instituto de Investigación Sanitaria (IIS), University of Zaragoza, and also with the CIBER de BioengenierÃna, Biomateriales y Nanomedicina (CIBER-BBN), Spain. E-mail: laguna@unizar.es

R. Jané

Institut de Bioenginyeria de Catalunya (IBEC), Dept. ESAII, Universitat PolitÃ̂́cnica de Catalunya, Barcelona, Spain and also with the CIBER de BioengenierÃna, Biomateriales y Nanomedicina (CIBERBBN). E-mail: rjane@ibecbarcelona.eu

S. Benito

Dept. of Emergency Medicine, Hospital de la Santa Creu i Sant Pau, Dept. of Medicine, Universitat Autónoma de Barcelona, Spain. E-mail: SBenito@santpau.cat

A. Bayes-Genis

Cardiology Dep., Hospital Universitari Germans Trias i Pujol, Dept. of Medicine, Universitat Autónoma de Barcelona, Spain. E-mail: abayesgenis@gmail.com

*Corresponding author

B.F. Giraldo

Dept. ESAII, EUETIB, Universitat PolitÃ̂́cnica de Catalunya, Barcelona; Institut de Bioenginyeria de Catalunya (IBEC), Barcelona, Spain and also with the CIBER de BioengenierÂna, Biomateriales y Nanomedicina (CIBER-BBN), Spain. E-mail: Beatriz.Giraldo@upc.edu 
$\mathrm{PB} / \mathrm{nPB}$ ) and 35 healthy subjects are studied. The results show that the maximal expiratory flow interval is shorter and with lower dispersion in CHF patients than in healthy subjects. The flow slopes are much steeper in CHF patients, especially for PB. Both inspiration and expiration durations are reduced in CHF patients, mostly for PB. Using the classification and regression tree technique, the most discriminant parameters are selected. For signals shorter than $1 \mathrm{~min}$, the time domain parameters produce better results than the spectral parameters, with accuracies for each classification of $82 / 78 \%, 89 / 85 \%$ and $91 / 89 \%$, respectively. It is concluded that morphologic analysis in the time domain is useful, especially when short signals are analyzed.

Keywords Chronic heart failure $\cdot$ respiratory pattern $\cdot$ periodic and non-periodic breathing $\cdot$ ensemble average.

\section{Introduction}

Chronic heart failure (CHF) is a growing epidemic in Western countries with increasing incidence and prevalence [5]. Despite important progress in recent decades, mortality remains high for patients with CHF. Moreover, established indexes such as New York Heart association (NYHA) functional class and the left ventricular ejection fraction (LVEF), associated with the laboratory values and medication use do not fully explain the mortality risk of patients with CHF and do not estimate an individual's prognosis [29], [19], [3]. CHF is associated with major abnormalities in the autonomic cardiovascular control, characterized by enhanced sympathetic nerve activity and cardiorespiratory disorder.

Breathing disorders are very common in CHF patients, usually manifested as a centrally-driven, rhythmic rise and fall in ventilation [26]. This type of breathing pattern, referred to as periodic breathing (PB), can be classified into ventilation with apnea, known as Cheyne-Stokes respiration, or without apnea [22], [18]. With a prevalence as high as $70 \%$ in CHF patients [27], the PB pattern has been established as a powerful predictor of poor prognosis in these patients [7] and increased mortality [12], especially in patients with Cheyne-Stokes respiration [30], [4].

Various parameters have been suggested for the characterization and identification of different breathing patterns [16], [31], [32], [35]. For example, the sleep-disordered breathing index is associated with an accelerated decline in cardiac function and increased morbidity and mortality [13], [14]. Sympathetic activity was found to increase with faster breathing rates and to decrease with higher tidal volumes in CHF patients. Some reports shown that the baroreflex sensitivity in CHF patients under basal conditions was lower than that of healthy subjects [1]. Short-term analysis of HRV has independent prognostic value of CHF patients: reduced low-frequency power during controlled breathing is a predictor of sudden death [17].

Several studies have reported that central sleep apnea is highly prevalent among patients with CHF [36], [34]. The continuous positive airway pressure treatment of obstructive sleep apnea in CHF patients demonstrated significant improvements in cardiac function and left ventricular systolic function, and attenuation of sympathetic nerve activity [15], [23]. 
We have previously characterized the respiratory pattern in CHF patients and healthy subjects using spectral parameters computed from the envelope of the respiratory flow signal [10]. In a subsequent study [11], we found that the correntropy function, using parameters based on the correntropy spectral density (CSD), was better suited for classification of respiratory patterns since this technique can be used to detect nonlinearities in the respiratory flow signal, which conventional techniques based on second-order statistics are unable to do [33], [20].

In general, differences in the spectral domain between $\mathrm{CHF}$ and healthy subjects are related to the modulation of the respiratory signal. The modulation may be characterized in the time domain by quantifying morphologic changes in the respiratory cycle, potentially offering the advantage of requiring shorter signals for analysis than required for computing the correntropy function.

The aim of this work is to study the morphology of the respiratory flow cycle in CHF patients with PB and non-periodic breathing (nPB). We analyze the evolution of morphology over time using a novel template-based technique. The accuracy of the proposed time domain parameters is evaluated for different signal lengths, and compared to CSD-based parameters [8].

\section{Methods}

The study of respiratory flow cycle morphology involves the following processing steps: segmentation and time alignment, computation of a respiratory cycle template from which a set of time domain parameters are extracted. On the other hand, CSDbased parameters are obtained to compare these results with those obtained with the morphology parameters. Finally, a statistical analysis is applied.

\subsection{Dataset}

Respiratory flow signals were recorded from 26 CHF patients (20 males; $65 \pm 9$ years) and 35 healthy volunteers (12 males; $27 \pm 7$ years) at Santa Creu i Sant Pau Hospital, Barcelona, Spain; see [10] for more details of the dataset. The study was approved by the local Ethics Committee. The signals were originally recorded at a sampling rate of $250 \mathrm{~Hz}$, for $15 \mathrm{~min}$, but, given that the frequency content of interest is below $1 \mathrm{~Hz}$, the signals were decimated to $10 \mathrm{~Hz}$ using zero-phase, antialiasing filtering.

All signals were analyzed visually by an experienced physician. According to clinical criteria, the PB patterns were visually identified by analyzing the waxing and waning of the respiratory flow signal. The CHF patients were divided into two groups: 8 patients with $\mathrm{PB}$ pattern ( 7 male; $71 \pm 7$ years) and 18 patients with nPB pattern (13 male; $63 \pm 9$ years).

The characteristics of the CHF patients and healthy subjects are shown in Table 1 . Figure 1 illustrates respiratory flow patterns observed in CHF patients (PB and nPB) and in a healthy subject. For the entire dataset with 61 subjects, the respiratory rate was found to range from 11.5 to 29.0 breaths/min. 
Table 1 Respiratory Rate (Mean \pm Standard Deviation) in CHF Patients and Healthy Subjects

\begin{tabular}{lcc} 
CHF & PB & nPB \\
& $(n=8)$ & $(n=18)$ \\
\hline$R_{f}$ (breaths/min) & $22.5 \pm 4.3$ & $18.4 \pm 2.2$ \\
$D_{I}(\mathrm{~s})$ & $0.97 \pm 0.20$ & $1.14 \pm 0.18$ \\
$D_{E}(\mathrm{~s})$ & $1.79 \pm 0.38$ & $2.16 \pm 0.32$ \\
\hline & $\mathrm{CHF}$ & Healthy subjects \\
& $(n=26)$ & $(n=35)$ \\
\hline$R_{f}$ (breaths/min) & $19.6 \pm 3.4$ & $15.5 \pm 3.7$ \\
$D_{I}(\mathrm{~s})$ & $1.09 \pm 0.20$ & $1.8 \pm 0.35$ \\
$D_{E}(\mathrm{~s})$ & $2.05 \pm 0.38$ & $2.3 \pm 0.64$ \\
\hline
\end{tabular}

$R_{f}$ : Respiratory rate; $D_{I}$ : Duration of inspiration; $D_{E}$ : Duration of expiration.

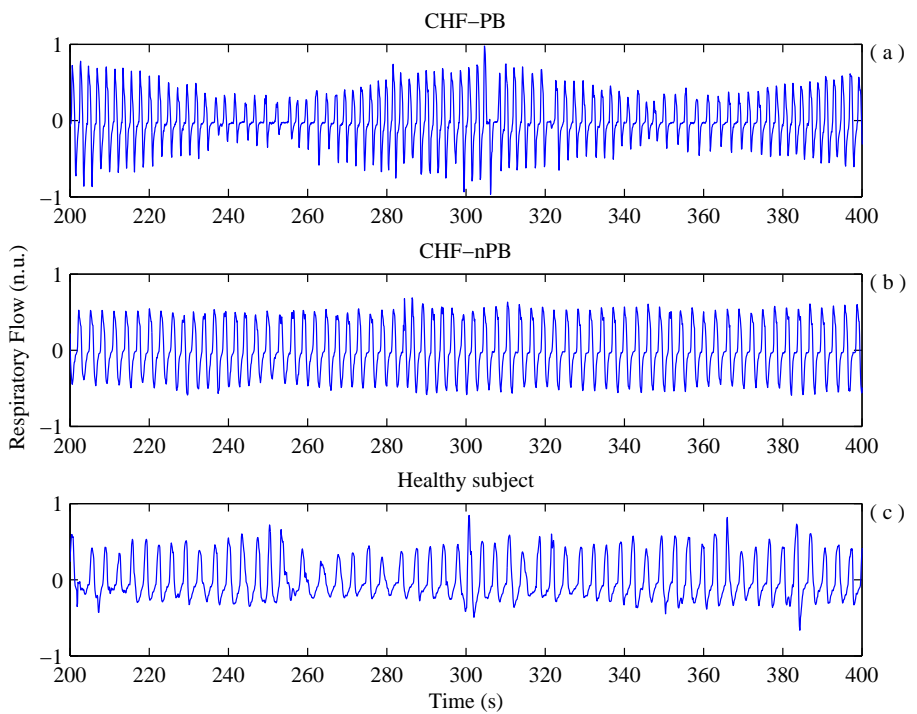

Fig. 1 Respiratory flow signals from (a) a CHF-PB patient, (b) a CHF-nPB patient, and (c) a healthy subject (n.u. denotes normalized units).

\subsection{Segmentation and Time Alignment}

The respiratory flow signal is analyzed using a sliding window of 30-s length (80\% overlap); this choice was based on the observation that the period length of PB ranges from 25 to $100 \mathrm{~s}$ [28]. The signal is normalized with respect to its largest absolute amplitude, and all cycles within the window are extracted using an algorithm that finds the zero-crossings of the respiratory flow signal. The resulting ensemble of successive respiratory cycles is represented by an $N \times M$ data matrix $\mathbf{X}$,

$$
\mathbf{X}=\left[\begin{array}{llll}
\mathbf{x}_{1} & \mathbf{x}_{2} & \cdots & \mathbf{x}_{M}
\end{array}\right]
$$


where each column $\mathbf{x}_{i}$ contains $N$ samples of the $i$ :th cycle, and $M$ is the total number of respiratory cycles.

To ensure that the signal ensemble is well-aligned, a novel eigenvalue-based method is employed which performs joint alignment of all signals; for more information on the method and its performance, see [9]. The method is based on the eigenvalue decomposition of the $N \times N$ sample correlation matrix as

$$
\hat{\mathbf{R}}_{x}=\frac{1}{M} \mathbf{X X}^{T}
$$

Interestingly, the ratio $\Lambda_{\boldsymbol{\theta}}$ between the largest eigenvalue $\lambda_{1}$ of $\hat{\mathbf{R}}_{x}$ and the sum of the remaining $N-1$ eigenvalues, i.e.,

$$
\Lambda_{\boldsymbol{\theta}}=\frac{\lambda_{1}}{\sum_{i=2}^{N} \lambda_{i}},
$$

can be interpreted as a signal-to-jitter-and-noise ratio [9]. Optimal alignment is obtained by finding those column shifts $\hat{\theta}_{1}, \ldots, \hat{\theta}_{M}$ which maximize the ratio $\Lambda_{\boldsymbol{\theta}}$. This is accomplished by shifting all columns symmetrically around their initial positions (corresponding to cycles aligned to the maximal inspiration), resulting in a combinatorial optimization problem. The alignment method was found to perform particularly well for small ensemble sizes, i.e., 10-20 cycles, which are analyzed in the present study.

\subsection{Respiratory flow cycle template}

A respiratory flow cycle template is calculated from the sliding window by computing the ensemble average of time-aligned respiratory flow cycles. The template is then characterized by a set of time domain parameters reflecting inspiratory and expiratory time intervals, maximal inspiratory and expiratory flow values, and down-/upward inspiratory and expiratory slopes, see Table 2 for a list of the parameters, and Fig. 2 for graphical representation.

Table 2 Time Domain Parameters Extracted from the Respiratory Flow Cycle Template

\begin{tabular}{cl} 
Parameter & Description \\
\hline$D_{I}[\mathrm{~s}]$ & Inspiration duration \\
$D_{E}[\mathrm{~s}]$ & Expiration duration \\
$M_{I}$ [n.u.] & Maximal inspiratory flow \\
$M_{E}$ [n.u.] & Maximal expiratory flow \\
$I_{I}$ [s] & Maximal inspiratory flow interval \\
$I_{E}$ [s] & Maximal expiratory flow interval \\
$S_{I 1}$ [n.u.] & Upward inspiratory slope \\
$S_{I 2}$ [n.u.] & Downward inspiratory slope \\
$S_{E 1}$ [n.u.] & Downward expiratory slope \\
$S_{E 2}$ [n.u.] & Upward expiratory slope \\
\hline
\end{tabular}




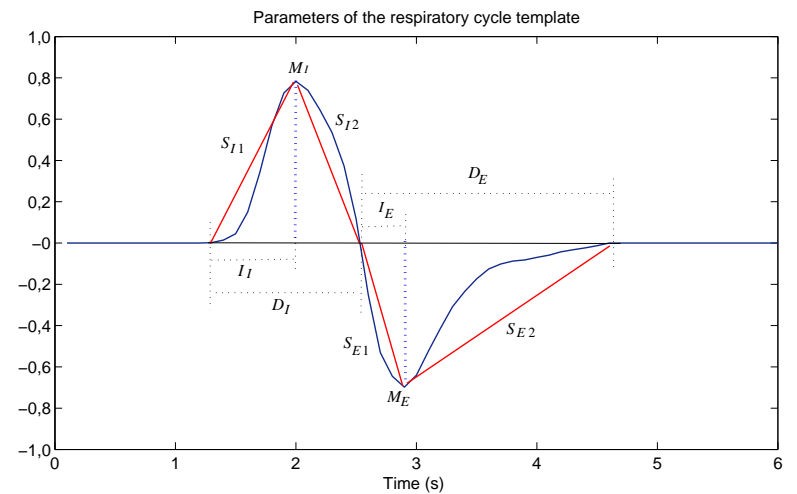

Fig. 2 Time domain parameters extracted from the respiratory flow cycle template.

Table 3 Parameters based on the Correntropy Spectral Density

\begin{tabular}{cl} 
Parameter & Description \\
\hline$P_{m}$ & Power of the modulation frequency band \\
$P_{r}$ & Power of the respiratory frequency band \\
$R$ & $P_{m} / P_{r}$ \\
$\bar{V}$ & Correntropy mean \\
\hline
\end{tabular}

\subsection{Spectral Parameters}

We showed that the CSD was particularly well-suited for the characterization of modulated breathing patterns [11]. In this study, the same characterization is applied but with signals much shorter than the 15 min used in [11]; here, the signal length ranges from 1 to $5 \mathrm{~min}$, incremented in steps of $30 \mathrm{~s}$. The CSD can be viewed as a generalization of the conventional power spectral density, but with better spectral resolution. The CSD-based parameters were derived in frequency bands centered around the peaks corresponding to the respiratory frequency and the modulation frequency, see Table 3.

\subsection{Statistical Analysis}

Statistical analysis was carried out using IBM SPSS software (v19). Differences between groups were tested by the Mann-Whitney U test. The mean and standard deviation of each time domain parameter computed every 30-seconds were evaluated as predictors for periodic breathing. The Classification and Regression Tree (CART) technique was used, together with leave-one-out cross-validation to identify the different breathing patterns. A $p$-value $<0.05$ was considered statistically significant. Parameters offering the best discriminatory power are presented in terms of accuracy, sensitivity, and specificity. 


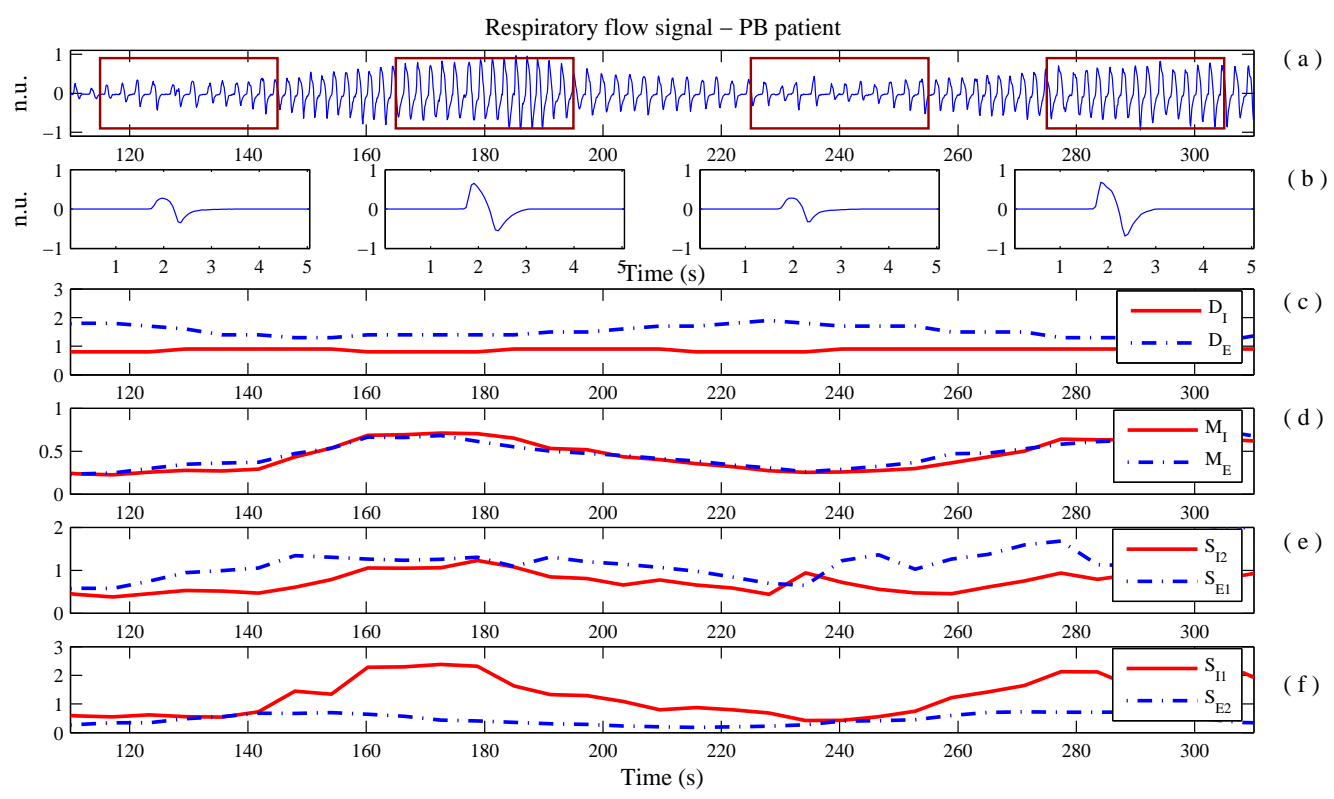

Fig. 3 Signals and trends of a CHF patient with PB: (a) The respiratory flow signal and (b) related templates determined in the four windows indicated in (a). The following trends, resulting from sliding window analysis, are displayed: (c) duration of inspiration $\left(D_{I}\right)$ and expiration $\left(D_{E}\right)$, (d) maximal inspiratory $\left(M_{I}\right)$ and expiratory $\left(M_{E}\right)$ flow value, $(\mathrm{e})$ downward inspiratory $\left(S_{I 2}\right)$ and expiratory $\left(S_{E 1}\right)$ slope, and (f) upward inspiratory $\left(S_{I 1}\right)$ and expiratory $\left(S_{E 2}\right)$ slope.

\section{Results}

3.1 Illustration of the Method

Examples of respiratory flow signals, respiratory cycle templates, and parameter trends are presented in Figs. 3, 4, and 5, for a patient with PB, a patient with nPB, and a healthy subject, respectively. It is noted from Fig. 3 that PB periodicity is well reflected by the trends of maximal inspiratory $\left(M_{I}\right)$, expiratory $\left(M_{E}\right)$ flow value, and duration of expiration $\left(D_{E}\right)$.

The upward and downward slopes of inspiration and expiration are represented by their respective mean values for each of the four groups, i.e., PB, nPB, CHF, and healthy, see Fig. 6. The respiratory flow cycle in CHF patients is compressed, especially for those with PB who exhibit the steepest slopes. Conversely, healthy subjects exhibit the least steep slopes, especially with respect to the upward expiratory slope $\left(S_{E 2}\right)$. 


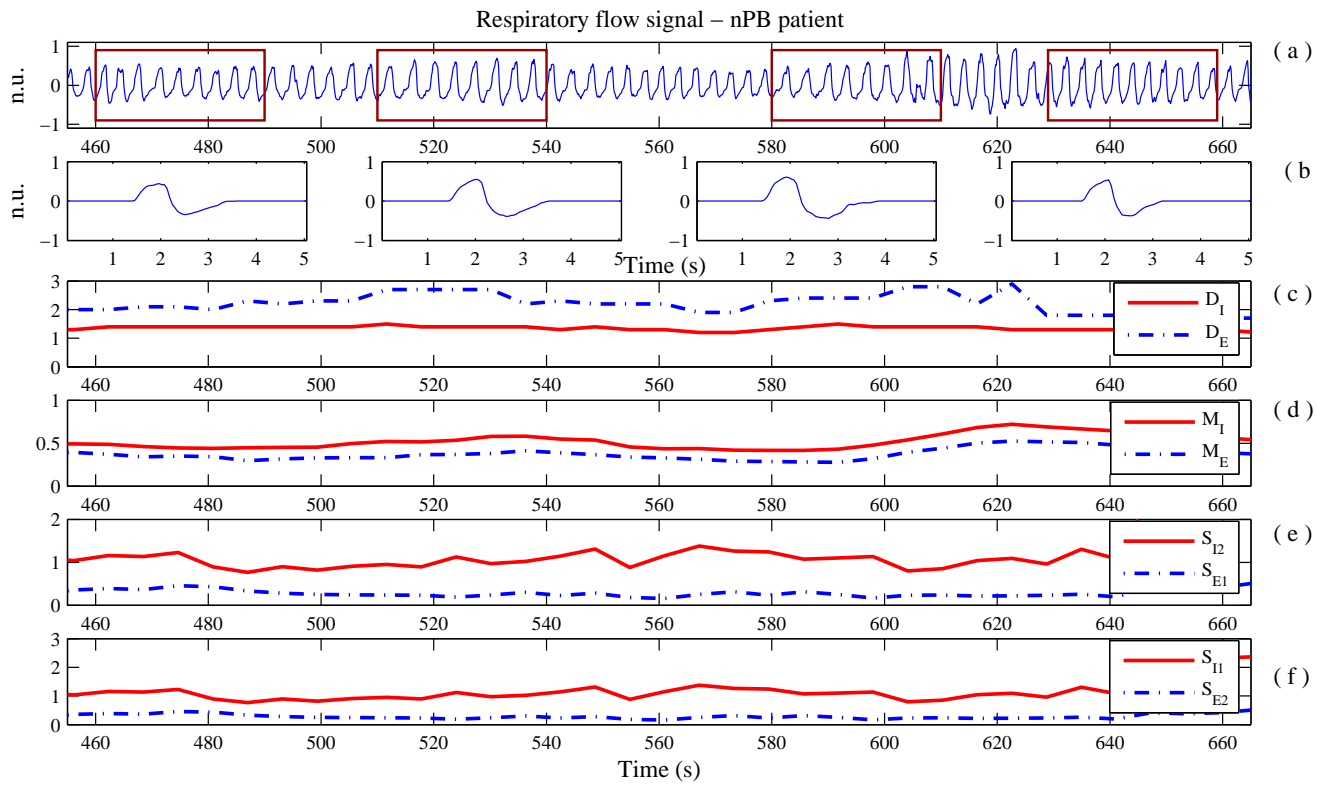

Fig. 4 Signals and trends of a CHF patient with nPB; see Fig. 3 for an explanation of (a)-(f).

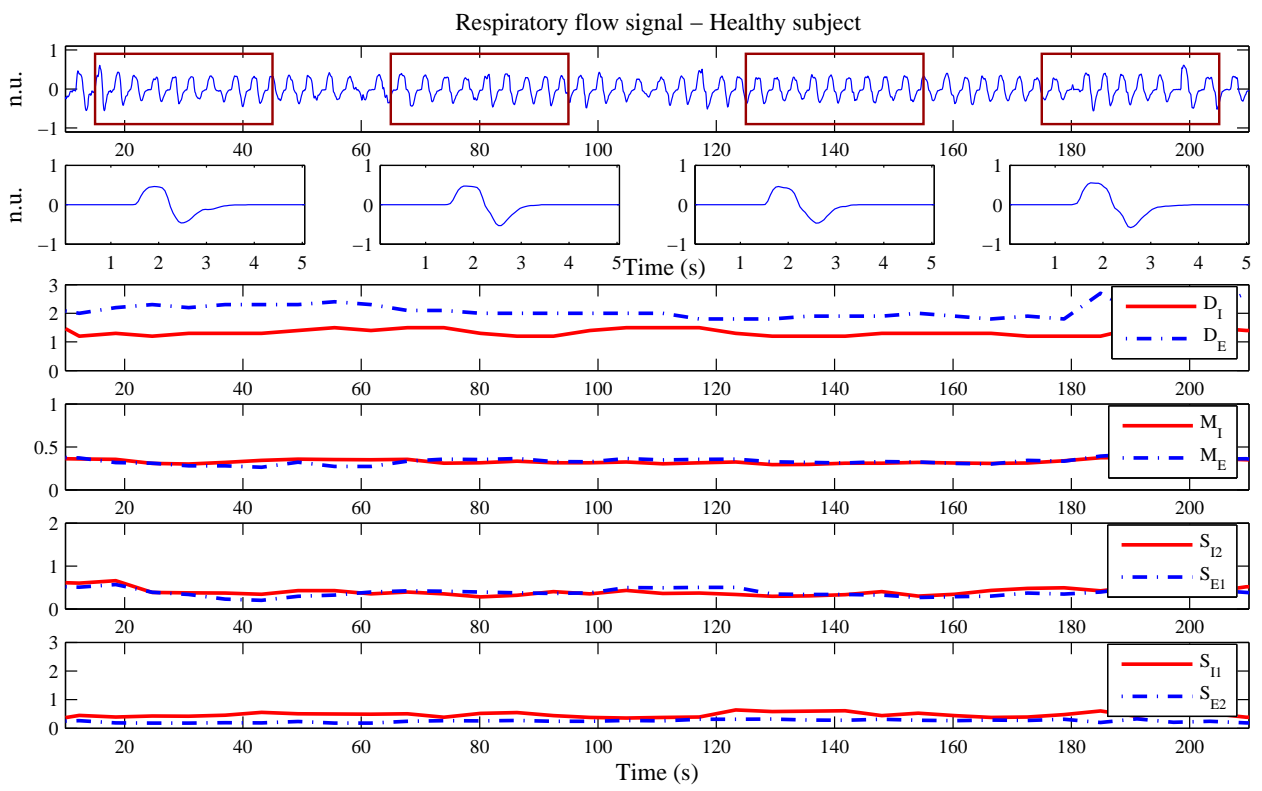

Fig. 5 Signals and trends of a healthy subject; see Fig. 3 for an explanation of (a)-(f). 

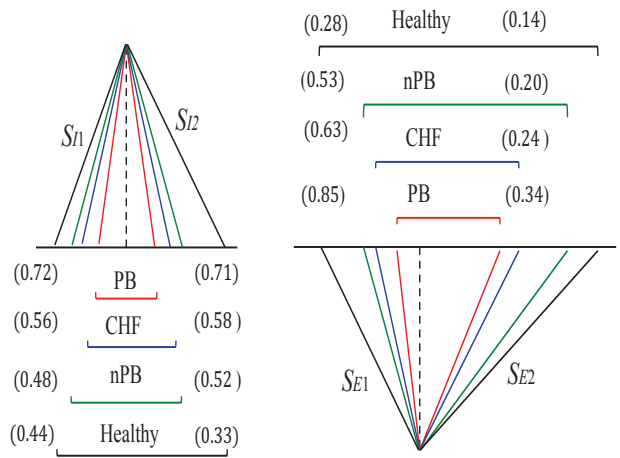

Fig. 6 Schematic representation of different slopes of the respiratory flow template cycle and their mean values.

3.2 Performance of the time domain parameters

Table 4 presents median, interquartile range, and $p$-value of the most statistically significant parameters when analyzing 15-min signals.

Table 4 Median and interquartile range of the most statistically significant parameters of the respiratory template cycle when comparing CHF patients, $\mathrm{PB}$ and nPB patients, and healthy subjects

\begin{tabular}{|c|c|c|c|c|c|c|c|}
\hline & CHF & PB & nPB & Healthy & $\begin{array}{c}p \text {-value } \\
C H F \text { vs. } H\end{array}$ & $\begin{array}{l}p \text {-value } \\
P B \text { vs. } n P B\end{array}$ & $\begin{array}{l}p \text {-value } \\
n P B \text { vs. } H\end{array}$ \\
\hline$M_{-} D_{I}$ & $1.20(0.30)$ & $1.11(0.28)$ & $1.23(0.30)$ & $1.5(0.36)$ & 0.0002 & n.s. & 0.005 \\
\hline$M_{-} D_{E}$ & $2.04(0.45)$ & $1.74(0.41)$ & $2.12(0.45)$ & $2.56(1.27)$ & 0.0003 & 0.016 & 0.009 \\
\hline$M_{-} I_{E}$ & $0.54(0.12)$ & $0.54(0.11)$ & $0.54(0.11)$ & $0.87(0.35)$ & 0.0004 & n.s. & 0.0003 \\
\hline$M S_{I 2}$ & $-0.68(0.41)$ & $-0.83(0.33)$ & $-0.66(0.27)$ & $-0.36(0.22)$ & 0.0004 & 0.041 & 0.004 \\
\hline$M \_S_{E 1}$ & $0.59(0.37)$ & $0.78(0.23)$ & $0.48(0.35)$ & $0.21(0.16)$ & 0.0005 & 0.009 & 0.0002 \\
\hline$S D_{-} D_{I}$ & $0.18(0.07)$ & $0.18(0.04)$ & $0.19(0.07)$ & $0.33(0.22)$ & 0.0004 & n.s. & 0.0005 \\
\hline$S D_{-} D_{E}$ & $0.27(0.15)$ & $0.21(0.09)$ & $0.30(0.20)$ & $0.47(0.46)$ & 0.0002 & n.s. & 0.008 \\
\hline$S D \_I_{E}$ & $0.10(0.04)$ & $0.10(0.02)$ & $0.09(0.05)$ & $0.21(0.15)$ & 0.0003 & n.s. & 0.0005 \\
\hline$S D \_S_{I 2}$ & $-0.28(0.23)$ & $-0.31(0.22)$ & $-0.27(0.24)$ & $-0.11(0.11)$ & 0.0005 & 0.026 & 0.006 \\
\hline$S D_{-} S_{E 1}$ & $-0.16(0.16)$ & $-0.26(0.18)$ & $-0.14(0.11)$ & $-0.08(0.06)$ & 0.0004 & 0.035 & 0.004 \\
\hline$S D \_S_{E 2}$ & $0.06(0.06)$ & $0.11(0.04)$ & $0.05(0.03)$ & $0.04(0.02)$ & 0.02 & 0.001 & n.s. \\
\hline
\end{tabular}

$M_{X X}$ : Mean value of each parameter; $S D_{X X}$ : Standard deviation of each parameter; n.s.: not significant $(p$-value $>0.05)$

$P B$ vs $n P B$ : The mean value of $D_{E}$ is lower in PB than in nPB patients $(p=0.009)$. All slopes $\left(S_{I 1}, S_{I 2}, S_{E 1}\right.$, and $\left.S_{E 2}\right)$ are steeper in PB patients than in nPB patients.

CHF patients vs healthy subjects: The mean values of $D_{I}$ and $D_{E}$ are lower in CHF patients than in healthy subjects. The maximal expiratory flow interval $I_{E}$ is shorter and with a lower dispersion in CHF patients than in healthy subjects. CHF patients present much steeper slopes $\left(S_{I 2}\right.$ and $\left.S_{E 1}\right)$ with a higher dispersion when compared 
Table 5 Accuracy (Acc), Sensitivity $\left(S_{E}\right)$ and Specificity $\left(S_{P}\right)$ obtained with the best parameter for each classification of 1-min signal length.

\begin{tabular}{lcccc} 
Classification & Parameter & $A c c$ & $S_{E}$ & $S_{P}$ \\
\hline Morphology parameters & & & \\
\hline PB vs. $n P B$ & $S D_{-} S_{E 2}$ & $82 \%$ & $75 \%$ & $94 \%$ \\
CHF vs. $H$ & $S D_{-} I_{E}$ & $89 \%$ & $85 \%$ & $91 \%$ \\
$n P B$ vs. $H$ & $M_{-} I_{E}$ & $91 \%$ & $84 \%$ & $91 \%$ \\
\hline CSD parameters & & & & \\
\hline PB vs. $n P B$ & $R$ & $78 \%$ & $88 \%$ & $74 \%$ \\
$C H F$ vs. $H$ & $\bar{V}$ & $85 \%$ & $100 \%$ & $74 \%$ \\
$n P B$ vs. $H$ & $\bar{V}$ & $89 \%$ & $100 \%$ & $68 \%$ \\
\hline
\end{tabular}

to healthy subjects. All parameters are significantly different with a $p<0.0005$, except the standard deviation of $S_{E 2}$ with $p=0.02$.

$n P B$ patients vs healthy subjects: The results show that $I_{E}$ is shorter and with a lower dispersion in nPB patients than in healthy subjects (both $p<0.0005$ ). Furthermore, it is shown that $S_{I 2}(p=0.004)$ and $S_{E 1}(p<0.0005)$ are steeper and with higher dispersion in $\mathrm{nPB}$ patients.

\subsection{Performance using different signal lengths}

The performance of the most discriminatory parameters $(p<0.01)$ is studied with respect to signal length, for ranges from 1 to $5 \mathrm{~min}$, being $S D \_S_{E 2}$ and $S D_{-} I_{E}$ in time domain and $R$ and $\bar{V}$ in the correntropy spectral domain. The accuracy, sensitivity, and specificity of these parameters are presented in Table 5 for 1-min signals (the minimal signal length used to calculate CSD values). When classifying PB vs nPB patients, the standard deviation of the upward expiratory slope $\left(S D \_S_{E 2}\right)$ has an accuracy of $82 \%$, and $R$ an accuracy of $78 \%$. When comparing CHF patients vs healthy subjects, $S D \_I_{E}$ has an accuracy of $89 \%$, whereas $\bar{V}$ has an accuracy of $85 \%$. When classifying nPB patients and healthy subjects, the mean of the maximal expiratory flow interval $\left(M_{-} I_{E}\right)$ correctly identifies the $91 \%$ of the subjects, and $\bar{V}$ the $89 \%$.

The accuracy of the most discriminatory parameters is evaluated for different signal lengths, see Figure 7. These results show that the time domain parameters perform slightly better than the CSD-based parameters for signals shorter than 1.5min. This difference is maintained for 5-min signals when classifying PB vs nPB patients with an accuracy of $96 \%\left(S D \_S_{E 2}\right)$ vs $93 \%(R)$. The CSD-based parameters perform slightly better than the time domain parameters for most signal lengths, when comparing CHF patients vs healthy subjects, and nPB patients vs healthy subjects. In these cases, the accuracies obtained with 5-min signals are $97 \%(\bar{V})$ vs $94 \%\left(S D_{-} I_{E}\right)$ in the first case, and $98 \%(\bar{V})$ vs $94 \%\left(M_{-} I_{E}\right)$ in the second case. 

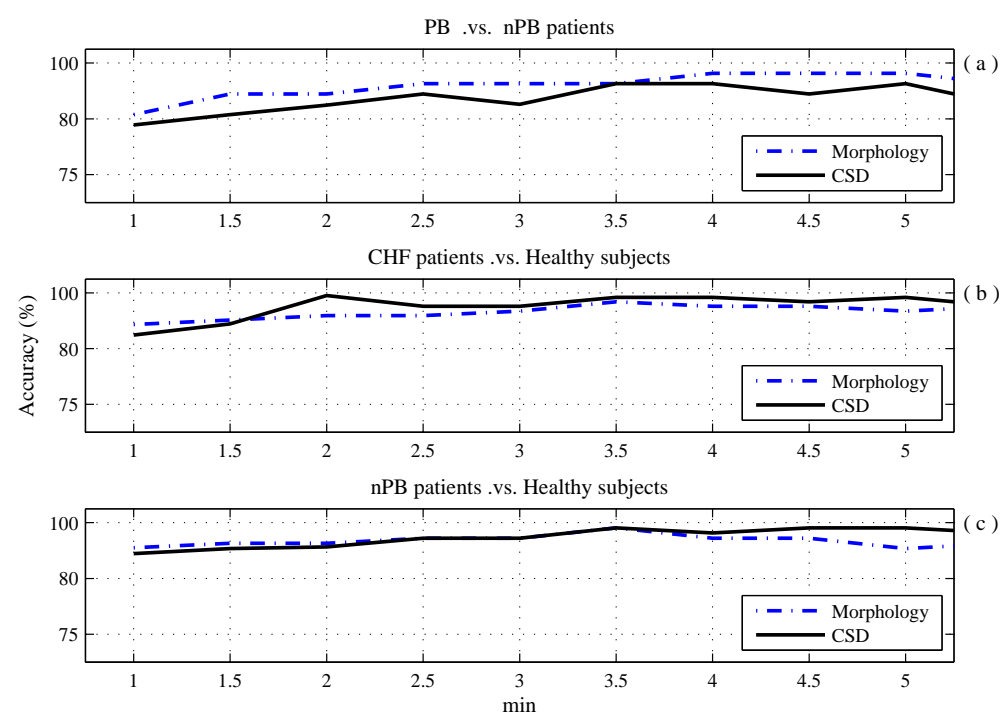

Fig. 7 Accuracy as a function of signal length, using the best parameters (see Table 5), when classifying (a) PB vs nPB patients, (b) CHF patients vs healthy subjects, and (c) nPB patients vs healthy subjects.

\section{Discussion}

In this study we have proposed a number of time domain parameters for morphologic characterization of the respiratory flow cycle in CHF patients with different conditions. A respiratory template cycle is calculated for every 30 -s respiratory flow signal segment. The time domain parameters, extracted from the template, provide statistically significant differences when comparing $\mathrm{CHF}$ patients ( $\mathrm{PB}$ and $\mathrm{nPB}$ ) and healthy subjects.

The estimated maximal expiratory flow interval is shorter and with a lower dispersion in CHF patients than in healthy subjects. CHF patients and foremost PB patients present a more compressed respiratory cycle. They show steeper slopes and lower inspiratory and expiratory times than healthy subjects. When classifying PB and nPB patients, all the slopes are steeper, and the standard deviation of the maximal inspiratory and expiratory flow value is much higher in PB than in $\mathrm{nPB}$ patients. This could be related to the periodicity of the respiratory pattern.

Periodic breathing pattern, frequently observed in patients with chronic heart failure, has been associated with increased risk of mortality. This pattern had been analyzed, mostly in relation with the sleep disorders [24], [35]; in healthy subjects, analyzing the relationship between the blood pressure and heart rate oscillations, through the tidal volume [21], and in the acclimatization to high altitude in healthy mountaineers [25], [6]. Despite advances in treatments of cardiovascular disease, the incidence and prevalence of heart failure continues to increase, which can be directly related to an increase in life span. In clinical practice, the CHF patients 
evolution is treated assessing indicators such as New York Heart Association (NYHA) classification, the percentage left ventricular ejection fraction (LVEF), and the level of natriuretic peptide (NT-proBNP) [2]. CHF patients normally have higher NYHA and ProBNP values, and lower LVEF percentages. Their diagnosis is based on tests whose results sometimes takes several days. Introducing additional information about the condition of the patient, using respiratory flow signal, could support the clinical decision and optimize the treatment.

In our previous studies, we characterized the respiratory pattern of CHF patients in the spectral domain using either the envelope of the respiratory flow signal [10], or the correntropy of the respiratory flow signal [11]. Correntropy was found to be particularly well-suited to characterize different breathing patterns in CHF patients and identify patientsâÁŹ condition. It was tested with 15-min of respiratory flow signals and provided an accuracy of $88.9 \%$ classifying PB vs nPB patients, and $95.2 \%$ classifying CHF patients vs healthy subjects.

Although CSD-based parameters perform slightly better with 5-min signals, shorter signals are needed for morphologic characterization to get significant parameters that identify $\mathrm{CHF}$ ( $\mathrm{PB}$ and $\mathrm{nPB}$ ) patients and healthy subjects. According to these results, 1-min signals could provide relevant information about patientâÁźs condition (see Fig. 7). Thus, a promising advantage of the present morphologic characterization is its ability to provide parameters in time domain, with a prognostic value using short respiratory flow signals.

An interesting conclusion is, that the dispersion of expiratory respiration evaluated through the upward slope and the maximal expiratory flow interval make the highest difference in the morphology of the respiratory flow cycle. These results are in concordance with the different slopes that describe the different groups of patients (Fig. 6).

In the clinical practice, the respiratory rate together with the peripheral pulse oximetry values are altered quickly with respiratory and cardiac diseases. CHF patient status is chronic and, the treatment every so often need to be reviewed and re-adapted. Changes in the respiratory flow cycle morphology can be a clear indicator of decompensation of these patients. The results of this study introduce parameters that can be very relevant in the decompensation of the patient, using shorter signals.

A better risk assessment, which includes breathing patterns in heart failure, is clinically of great value as it more accurately identifies patients with $\mathrm{CHF}$ at increased risk of re-hospitalization and/or death who could then be targeted for more intensive monitoring and personalized treatment. Once these results are clinically validated, the incorporation of the respiratory patterns into clinical practice for the prediction of death could be accomplished.

Limitations of the study. A limitation of this study is that the number of patients studied is small, especially the group of patients with periodic breathing. Another limitation is the difference between ages of the healthy group and CHF patients group. 


\section{Conclusions}

Based on a respiratory template cycle, obtained with a novel time alignment method, various parameters such as inspiratory and expiratory durations, maximal expiratory flow interval and slopes are found to offer good discrimination between respiratory patterns in $\mathrm{CHF}$ patients ( $\mathrm{PB}$ and $\mathrm{nPB}$ ) and healthy subjects. Maximal expiratory flow interval occurs earlier with higher slopes, and reduced inspiratory and expiratory duration, in CHF patients and foremost in PB patients than in healthy subjects. The results suggest that the analysis of the respiratory flow cycle morphology is a promising approach to study respiratory patterns in CHF patients, especially when dealing with short length signals.

Acknowledgements This work was supported in part by the Spanish GovernmentâĂŹs Ministerio de Economía y Competitividad under grant TEC2010-21703-C03.

\section{References}

1. Bernardi, L., Porta, C., Spicuzza, L., Bellwon, J., Spadacini, G., Frey, A., Yeung, L., Sanderson, J.E. Pedretti, R., Tramarin, R.: Slow breathing increases arterial baroreflex sensitivity in patients with chronic heart failure. Circulation 105, 143-145 (2002)

2. Bhardwaj, A., Rehman, S., Mohammed, A., Gaggin, H., Barajas, L., Barajas, J., Moore, S., Sullivan, D., Januzzi, J.: Quality of life and chronic heart failure therapy guided by natriuretic peptides: Results from the probnp outpatient tailored chronic heart failure therapy (protect) study. Am Heart J 164, 793-799.e1. (2012)

3. Bouvy, M., Heerdink, E., Leufkens, H., Hoes, A.: Predicting mortality in patients with heart failure: a pragmatic approach. Heart 89(6), 605-609 (2003)

4. Corra, U., Pistono, M., Mezzani, A., Braghiroli, A., Giordano, A., Lanfranchi, P., Bosimini, E., Gnemmi, M., Giannuzzi, P.: Sleep and Exertional Periodic Breathing in Chronic Heart Failure: Prognostic Importance and Interdependence. Circulation 113(1), 44-50 (2006)

5. Dickstein, K., Cohen-Solal, A., Filippatos, G., McMurray, J., Ponikowski, P., Poole-Wilson, P., Stromberg, A., van Veldhuisen, D., Atar, D., Hoes, A., Keren, A., Mebazaa, A., Nieminen, M., Priori, S., Swedberg, K.: Esc guidelines for the diagnosis and treatment of acute and chronic heart failure 2008: the task force for the diagnosis and treatment of acute and chronic heart failure 2008 of the european society of cardiology. Eur Heart J. 29(19), 2388-2442 (2008)

6. Fernandez, H., Pattyn, N., Mairesse, O., Meeusen, R., Mcdonald-Nethercott, E., Neyt, X.: One year monitoring of nocturnal periodic breathing at the antarctic pole. Sleep Medicine 14(Supplement 1), e119 (2013)

7. Francis, D.P., Willson, K., Davies, L.C., Coats, A.J., Piepoli, M.: Quantitative general theory for periodic breathing in chronic heart failure and its clinical implications. Circulation 102(18), 2214 $2221(2000)$

8. Garde, A., Giraldo, B., Sörnmo, L., Jané, R.: Analysis of the respiratory flow cycle morphology in chronic heart failure patients applying principal components analysis. In: Proc. IEEE Conf. Eng. Med. Biol., pp. 1725-1728 (2011)

9. Garde, A., Laguna, P., Giraldo, B., Jané, R., Sörnmo, L.: Ensemble-based time alignment of biomedical signals. In: 7th International Workshop on Biosignal Interpretation âĂŞ BSI2012, Como, Italy, pp. 1-4 (2012)

10. Garde, A., Sörnmo, L., Jané, R., Giraldo, B.: Breathing pattern characterization in chronic heart failure patients using respiratory flow signal. Ann. Biomed. Eng. 38(12), 3572-3580 (2010)

11. Garde, A., Sörnmo, L., Jané, R., Giraldo, B.: Correntropy-based spectral characterization of respiratory patterns in patients with chronic heart failure. IEEE Trans. Biomed. Eng. 57(8), 1964-1972 (2010)

12. Guazzi, M., Raimondo, R., Vicenzi, M., Arena, R., Proserpio, C., Sarzi Braga, S., Pedretti, R.: Exercise Oscillatory Ventilation May Predict Sudden Cardiac Death in Heart Failure Patients. J Am Coll Cardiol 50(4), 299-308 (2007) 
13. Hanly, P.J., Zuberi-Khokhar, N.S.: Increased mortality associated with Cheyne-Stokes respiration in patients with congestive heart failure. Am. J Resp. Crit. Care Med. 153(1), 272-276 (1996)

14. Javaheri, S., Parker, T.J., Liming, J.D., Corbett, W.S., Nishiyama, H., Wexler, L., Roselle, G.A.: Sleep apnea in 81 ambulatory male patients with stable heart failure. Types and their prevalences, consequences, and presentations. Circulation 97(21), 2154-2159 (1998)

15. Kaneko, Y., Floras, J.S., Usui, K., Plante, J., Tkacova, R., Kubo, T., Ando, S., Bradley, T.D.: Cardiovascular effects of continuous positive airway pressure in patients with heart failure and obstructive sleep apnea. New England J. Med. 348(13), 1233-1241 (2003)

16. Kesper, K., Canisius, S., Penzel, T., Ploch, T., Cassel, W.: Ecg signal analysis for the assessment of sleep-disordered breathing and sleep pattern. Med Bio Eng Comput 50, 135-144 (2012)

17. La-Rovere, M.T., Pinna, G.D., Maestri, R., Mortara, A., Capomolla, S., Febo, O., Ferrari, R., Franchini, M., Gnemmi, M., Opasich, C., Riccardi, P.G., Traversi, E., Cobelli, F.: Short-term heart rate variability strongly predicts sudden cardiac death in chronic heart failure patients. Circulation 107, 565-570 (2003)

18. Lanfranchi, P.A., Braghiroli, A., Bosimini, E., Mazzuero, G., Colombo, R., Donner, C.F., Giannuzzi, P.: Prognostic value of nocturnal Cheyne-Stokes respiration in chronic heart failure. Circulation 99(11), 1435-1440 (1999)

19. Levy, W., Mozaffarian, D., Linker, D., Sutradhar, S., Anker, S., Cropp, A., Anand, I., Maggioni, A., Burton, P., Sullivan, M., Pitt, B., Poole-Wilson, P., Mann, D., Packer, M.: The seattle heart failure model: prediction of survival in heart failure. Circulation 113(11), 1424-1433 (2006)

20. Liu, W., Pokharel, P.P., Principe, J.C.: Correntropy: properties and applications in non-Gaussian signal processing. IEEE Trans. Signal Proc. 55(11), 5286-5298 (2007)

21. Lorenzi-Filho, G., Dajani, H., Leung, R., Floras, J., douglas, T.: Entrainment of blood pressure and heart rate oscillations by periodic breathing. Am J Respir Crit Care Med 159, 1147-1154 (1999)

22. Lorenzi-Filho, G., Genta, P.R., Figueiredo, A.C., Inoue, D.: Cheyne-Stokes respiration in patients with congestive heart failure: causes and consequences. Clinics (Sao Paulo, Brazil) 60(4), 333-344 (2005)

23. Mansfield, D.R., Gollogly, N.C., Kaye, D.M., Richardson, M., Bergin, P., Naughton, M.T.: Controlled trial of continuous positive airway pressure in obstructive sleep apnea and heart failure. Am. J. Resp. Crit. Care Med. 169(3), 361-366 (2004)

24. Millar, T., Hanly, P., Kryger, M.: Short technical note: Quantifiaction of periodic breathong: preliminary studies. Sleep 15(4), 364-370 (1992)

25. Nussbaumer-Ochsner, Y., Ursprung, J., Siebenmann, C., Maggiorini, M., Bloch, K.: Effect of shortterm acclimatization to high altitude on sleep and nocturnal breathing. Sleep 35(3), 419-423 (2012)

26. Pinna, G., Maestri, R., Robbi, E., Rovere, M.L.: Periodic breathing and state instability during supine laboratory recordings in chronic heart failure patients. In: Proc. IEEE Conf. Eng. Med. Biol., pp. 5398-5401 (2008)

27. Pinna, G.D., Maestri, R., Mortara, A., Johnson, P., Witkowski, T., Ponikowski, P., Andrews, D., Capomolla, S., La Rovere, M.T., Sleight, P.: Nocturnal periodic breathing is an independent predictor of cardiac death and multiple hospital admissions in heart failure. In: Proc. Comput. Cardiol., vol. 33, pp. 837-840 (2006)

28. Pinna, G.D., Maestri, R., Mortara, A., La Rovere, M.T., Fanfulla, F., Sleight, P.: Periodic breathing in heart failure patients: testing the hypothesis of instability of the chemoreflex loop. J. Appl. Physiol. 89(6), 2147-2157 (2000)

29. Pocock, S., Wang, D., Pfeffer, M., Yusuf, S., McMurray, J., Swedberg, K., Ostergren, J., Michelson, E., Pieper, K., Granger, C.: Predictors of mortality and morbidity in patients with chronic heart failure. Eur Heart J. 27(1), 65-75 (2006)

30. Poletti, R., Passino, C., Giannoni, A., Zyw, L., Prontera, C., Bramanti, F., Clerico, A., Piepoli, M., Emdin, M.: Risk factors and prognostic value of daytime cheyne-stokes respiration in chronic heart failure patients. International Journal of Cardiology 137(1), 47 - 53 (2008)

31. Ponikowski, P., Anker, S.D., Chua, T.P., Francis, D., Banasiak, W., Poole-Wilson, P.A., Coats, A.J., Piepoli, M.: Oscillatory breathing patterns during wakefulness in patients with chronic heart failure: clinical implications and role of augmented peripheral chemosensitivity. Circulation 100(24), 24182424 (1999)

32. Ribeiro, J.P.: Periodic breathing in heart failure: bridging the gap between the sleep laboratory and the exercise laboratory. Circulation 113(1), 9-10 (2006)

33. Santamaria, I., Pokharel, P.P., Principe, J.C.: Generalized correlation function: definition, properties, and application to blind equalization. IEEE Trans. Signal Proc. 54(6), 2187-2197 (2006) 
34. Solin, P., Roebuck, T., Johns, D., Walters, E., Naughton, M.: Peripheral and central ventilatory responses in central sleep apnea with and without congestive heart failure. Am J Respir Crit Care Med 162, 2194âĂŞ2200 (2000)

35. Vazir, A., Dayer, M., Hastings, P.C., McIntyre, H.F., Henein, M.Y., Poole-Wilson, P.A., Cowie, M.R., Morrell, M.J., Simonds, A.K.: Can heart rate variation rule out sleep-disordered breathing in heart failure? Eur. Resp. J. 27(3), 571-577 (2006)

36. Yamashiro, S.: Non-linear dynamics of human periodic breathing and implications for sleep apnea therapy. Med Bio Eng Comput 45, 345âĂŞ356 (2007) 